\title{
Low Dose Indomethacin in the Outpatient Treatment of COVID-19 in Kidney Transplant Recipients-A Case Series
}

\author{
Arumugham Kanakaraj ${ }^{1}$, Rajan Ravichandran² \\ ${ }^{1}$ Consultant Nephrologist, Chennai, India \\ ${ }^{2}$ Head of Department, Nephrology, MIOT International, Chennai, India \\ Email: a.kanakaraj.ka@gmail.com
}

How to cite this paper: Kanakaraj, A. and Ravichandran, R. (2020) Low Dose Indomethacin in the Outpatient Treatment of COVID-19 in Kidney Transplant Recipients-A Case Series. Open Access Library Journal, 7: e6860.

https://doi.org/10.4236/oalib.1106860

Received: September 24, 2020

Accepted: October 26, 2020

Published: October 29, 2020

Copyright $\odot 2020$ by author(s) and Open Access Library Inc.

This work is licensed under the Creative Commons Attribution International License (CC BY 4.0).

http://creativecommons.org/licenses/by/4.0/

\begin{abstract}
The pandemic caused by the coronavirus SARS-CoV-2 has affected millions of people around the world. There are no definitive treatments available to date, which has led to the use of several repurposed drugs. Indomethacin is an effective non-steroidal anti-inflammatory drug, which has played an important role in the treatment of rheumatological conditions and cytokine storms, in the past. Coronavirus disease 2019 (COVID-19) causes cytokine storm and multiorgan dysfunction resulting in high mortality in those affected. Kidney transplant recipients are susceptible to COVID-19, which has caused a higher mortality than the general population, due to the immunosuppressed state. Twelve kidney transplant recipients under regular follow up at our hospital, consulted over the telephone, if they had features of COVID-19. Low dose indomethacin in this group of patients was given in those who were assessed to have mild disease and could be managed at home. Four of twelve patients were admitted to hospital due to their worsening symptoms. However, none of them died and all the 12 patients made good recovery at the end of 4 weeks. This has resulted in not only treating their symptoms, but probably also prevented more hospitalizations by avoiding or attenuating a cytokine storm in them. Indomethacin could prove to be a useful drug in the treatment of mild COVID-19, in view of its easy availability, low cost and minimal side effects.
\end{abstract}

\section{Subject Areas}

Nephrology

\section{Keywords}

Coronavirus Disease (COVID-19), Kidney Transplant Recipient, 
Indomethacin

\section{Introduction}

Coronavirus disease 2019 (COVID-19) is a rapidly spreading pandemic, which originated in Wuhan, China, followed by an increasing number of cases throughout the world. India is one of the worst affected countries, having a burden of over four million cases and seventy thousand deaths, by the first week of September 2020 (https://www.mygov.in/covid-19). The protean manifestations of this disease in different subgroups of the population combined with limited definitive treatments available have been a challenge to the treating physicians. Patients with chronic kidney disease, particularly kidney transplant recipients are susceptible to COVID-19 due to immunosuppression [1] [2]. Mortality in COVID-19 has been attributed to cytokine storm resulting in acute respiratory distress syndrome (ARDS) and multi-organ dysfunction. Various pharmacological therapies have been evaluated to manage this disease. Several repurposed drugs were also evaluated, in an effort to find a cure. Indomethacin is one such drug which may be useful in this setting. It is a potent non-steroidal anti inflammatory drug which has been used for many decades in the treatment of many rheumatological conditions as well as in the management of cytokine storm. We describe the clinical features and outcomes of twelve kidney transplant recipients who received indomethacin for the treatment of mild COVID-19.

\section{Subjects and Methods}

During the pandemic, from the 1 st of June to 31st of July 2020, kidney transplant recipients were advised to contact us over the telephone to report any symptoms suggestive of COVID-19. Twelve kidney transplant recipients, who underwent transplantation at our centre and under regular follow up were included in this study. The inclusion criteria were 1) kidney transplant recipients with mild symptoms of COVID-19, like low grade fever, cough, sore throat, mild breathlessness, myalgia, anorexia and occasional diarrhea or vomiting. 2) able to monitor temperature and oxygen saturation by a pulse oximeter at home every 4 hours. 3) to report on a daily basis of their symptoms, temperature and oxygen saturation. The exclusion criteria were a) moderate or severe symptoms like high grade fever with chills, severe breathlessness, persistent vomiting, diarrhea or extreme tiredness. b) oxygen saturation $<93 \%$ by pulse oximetry. c) inability to manage at home by the patient or caregiver. Patients were advised to do nasopharyngeal swab RT-PCR test for SARS-CoV 2, when they developed symptoms of COVID-19. They were treated with Indomethacin (immediate release) $25 \mathrm{mg}$ twice a day for 5 days, along with omeprazole $20 \mathrm{mg}$ twice a day, if the RT-PCR was positive. They were not admitted, if they had mild symptoms. Immunosup- 
pressants were not altered. Their condition was assessed everyday over the phone, including temperature and oxygen saturation. Admission to hospital was advised if they developed worsening of their symptoms or drop in saturation. The outcome and change in their clinical status was noted.

\section{Results}

Twelve kidney transplant recipients developed mild COVID-19 disease. Patients who had features of moderate or severe disease like severe breathlessness or oxygen saturation less than $93 \%$ were advised to get admitted to the hospital. Patients who had features of mild illness were treated as outpatients, after nasopharyngeal swab RT-PCR test for SARS-CoV2. They were given Indomethacin $25 \mathrm{mg}$ (immediate release) twice a day and managed as described earlier. The clinical characteristics are described below (Table $1 \&$ Table 2).

The median age of the patients was 40.5 years (range 30 - 64). Eleven of them (91.6\%) were men. The majority of them had common comorbidities like diabetes mellitus and systemic hypertension (50\% and 100\% respectively). Two of the patients had established coronary artery disease, one had a coronary stent and the other had undergone coronary artery bypass graft several years earlier. Interestingly only one patient had underlying asthma. He also had previous venous thromboembolism and was already on long term warfarin. One patient had myelodysplastic syndrome on long term hydroxyurea and another patient

Table 1. Characteristics of kidney transplant recipients with COVID-19 $(n=12)$.

\begin{tabular}{|c|c|}
\hline & Median (range) or $\mathrm{n}(\%)$ \\
\hline Age, years & $40.5(30-64)$ \\
\hline Men & $11(91.6 \%)$ \\
\hline Months after transplant & $73(7-126)$ \\
\hline Diabetes mellitus & $6(50 \%)$ \\
\hline Hypertension & $12(100 \%)$ \\
\hline Ischemic heart disease & $2(16.6 \%)$ \\
\hline Lung disease & $1(8.3 \%)$ \\
\hline Prior malignancy & $2(16.6 \%)$ \\
\hline Weight, $\mathrm{kg}$ & $76.05(55.1-101)$ \\
\hline Fever, days & $2(0-6)$ \\
\hline Cough & $4(33.3 \%)$ \\
\hline Breathlessness & $3(25 \%)$ \\
\hline Lethargy & $11(91.6 \%)$ \\
\hline Myalgia & $8(66.6 \%)$ \\
\hline Anorexia & $4(33.3 \%)$ \\
\hline Baseline S.Cr, mg/dl & $1.45(0.7-3)$ \\
\hline S.Cr, mg/dl, at 4 weeks & $1.45(0.7-2.9)$ \\
\hline Time to recovery, days & $5(2-28)$ \\
\hline
\end{tabular}


Table 2. Individual patient characteristics of kidney transplant recipients with COVID-19.

\begin{tabular}{|c|c|c|c|c|c|c|c|c|c|c|c|c|}
\hline & 1 & 2 & 3 & 4 & 5 & 6 & 7 & 8 & 9 & 10 & 11 & 12 \\
\hline Age, years & 30 & 46 & 39 & 60 & 37 & 30 & 40 & 41 & 64 & 40 & 52 & 52 \\
\hline Sex & M & M & M & M & $\mathrm{F}$ & M & M & M & M & M & M & M \\
\hline Diabetes & No & No & Yes & Yes & No & No & No & No & Yes & Yes & Yes & Yes \\
\hline Hypertension & Yes & Yes & Yes & Yes & Yes & Yes & Yes & Yes & Yes & Yes & Yes & Yes \\
\hline IHD & No & No & No & No & No & No & No & No & Yes & No & No & Yes \\
\hline Lung Disease & No & No & Yes & No & No & No & No & No & No & No & No & No \\
\hline Prior malignancy & No & No & No & No & Yes & No & No & No & Yes & No & No & No \\
\hline CKD cause & CGN & $\operatorname{IgA}$ & CGN & $\mathrm{DN}$ & $\operatorname{Ig} \mathrm{A}$ & $\mathrm{RN}$ & $\mathrm{RN}$ & $\mathrm{CIN}$ & $\mathrm{DN}$ & $\operatorname{IgA}$ & $\mathrm{DN}$ & $\mathrm{DN}$ \\
\hline $\begin{array}{l}\text { Months after } \\
\text { transplant }\end{array}$ & 42 & 67 & 79 & 102 & 100 & 61 & 104 & 52 & 105 & 126 & 47 & 7 \\
\hline Type of transplant & $\mathrm{L}$ & $\mathrm{L}$ & $\mathrm{DD}$ & $\mathrm{L}$ & $\mathrm{ABOi}$ & $\mathrm{DD}$ & $\mathrm{L}$ & $\mathrm{L}$ & $\mathrm{L}$ & $\mathrm{L}$ & $\mathrm{L}$ & $\mathrm{L}$ \\
\hline Fever & Yes & Yes & No & Yes & Yes & Yes & Yes & Yes & Yes & No & No & Yes \\
\hline Cough & Yes & No & No & Yes & Yes & No & No & No & No & Yes & No & No \\
\hline Breathlessness & Yes & No & No & No & Yes & No & No & No & No & Yes & No & No \\
\hline Myalgia & No & No & Yes & No & Yes & Yes & Yes & Yes & Yes & No & Yes & Yes \\
\hline Lethargy & Yes & No & Yes & Yes & Yes & Yes & Yes & Yes & Yes & Yes & Yes & Yes \\
\hline Anorexia & No & No & No & Yes & Yes & No & Yes & No & No & No & Yes & No \\
\hline Weight, kg & 65.2 & 75 & 85.9 & 55.1 & 99.3 & 77.2 & 101 & 75.3 & 62.3 & 77.5 & 76.5 & 75.6 \\
\hline Baseline S.cr mg/dl & 1 & 1.1 & 0.8 & 0.7 & 2 & 2.8 & 3 & 1.1 & 1.4 & 2.5 & 2 & 1.5 \\
\hline Recovery, days & 3 & 3 & 10 & 5 & 15 & 2 & 2 & 10 & 14 & 28 & 5 & 5 \\
\hline Immunosuppressants & PTM & PTM & PTA & PTM & $\mathrm{PM}$ & PTA & PCM & PTM & PTA & PTM & PTA & PTM \\
\hline ACEi/ARB & Yes & No & No & No & No & No & No & No & No & Yes & No & No \\
\hline Hospitalization & No & No & Yes & No & No & No & No & Yes & Yes & Yes & No & No \\
\hline $\begin{array}{l}\text { S.Cr.mg/dl after } 4 \\
\text { weeks }\end{array}$ & 1 & 1.4 & 0.8 & 0.7 & 2.1 & 2.7 & 2 & 1.3 & 1.4 & 2.9 & 1.5 & 1.5 \\
\hline
\end{tabular}

IHD-Ischemic heart disease, P-prednisolone, T-tacrolimus, C-cyclosporine, M-Mycophenolate, A-Azathioprine, CGN-chronic glomerulonephritis, CIN-chronic interstitial nephritis, DN-diabetic nephropathy, RN-reflux nephropathy, L-living donor, DD-deceased donor, ABOi-ABO-incompatible kidney transplantation.

had undergone radical nephrectomy for renal cell carcinoma of a native kidney after transplantation. Both of them were regularly monitored for cancer recurrence and were found to be free from cancer. The underlying cause for chronic kidney disease included diabetic nephropathy $(\mathrm{n}=4)$, IgA nephropathy $(\mathrm{n}=3)$, chronic glomerulonephritis and reflux nephropathy $(\mathrm{n}=2$ each) and chronic interstitial nephritis $(n=1)$. Two of them underwent deceased donor kidney transplantation and one underwent $\mathrm{ABO}$-incompatible kidney transplantation. The majority ( 9 out of 12) of them underwent living donor kidney transplantation. The median duration from the transplantation to the onset of COVID-19 was 73 months (range 7 - 126 months). Fever was a predominant symptom in 9 of 12 patients with a median duration of 2 days (range $0-6$ days). Cough and breathlessness were present only in $33.3 \%$ and $25 \%$ respectively. More common 
presenting symptoms were myalgia and tiredness occurring in $66.6 \%$ and $91.6 \%$ respectively. Other less common symptoms were anorexia (33.3\%), abdominal pain, nausea, vomiting and diarrhea ( $8.3 \%$ each). Many of the transplant recipients were overweight, and the median weight was $76.05 \mathrm{~kg}$ (range 55.1 - 101 $\mathrm{kg}$ ). The median baseline serum creatinine was $1.45 \mathrm{mg} / \mathrm{dl}$ (range $0.7-3 \mathrm{mg} / \mathrm{dl}$ ). Except one patient, all others were on prednisolone and a calcineurin inhibitor. Four of them were on azathioprine and the remaining 8 of them were on mycophenolate mofetil or mycophenolate sodium. Two of them were on ACE inhibitors or angiotensin receptor blockers, which were continued. As mentioned earlier the immunosuppressants were not altered during the illness, if they were treated as an outpatient. Hospitalized patients received intravenous methylprednisolone 40 - $60 \mathrm{mg}$ twice a day, until discharge along with remdesivir, as per protocol. Calcineurin inhibitor dose was reduced and anti metabolite was stopped. None of the 4 patients required mechanical ventilation. Indomethacin was given for 5 days in 10 patients, and 2 of them received it for 10 days, due to persistent symptoms. None of the patients reported any significant side effects. The median time to recovery was 5 days (range 2 - 28 days). Serum creatinine at the end of 4 weeks did not differ significantly from the baseline. The median serum creatinine was $1.45 \mathrm{mg} / \mathrm{dl}$ (range 0.7 - 2.9). None of the 12 patients relapsed clinically by 4 weeks, and RT-PCR for SARS-CoV2 was not repeated.

\section{Discussion}

Kidney transplant recipients are susceptible to bacterial, viral and fungal infections due to their immunosuppressed state. Several viruses including cytomegalovirus, herpes and influenza have caused serious infections in them [3]. The current pandemic of COVID-19 has also affected the kidney transplant recipients, similar to the general population. Guidelines from different societies are available to treat these patients [4] [5].

Indomethacin has been used for many rheumatological diseases for several decades. Interest in this molecule in the treatment of coronavirus disease was triggered by a study by Amici et al., in 2006. Indomethacin at cytoprotective doses was found to have a potent direct in vitro antiviral activity against the coronaviruses SARS-CoV and canine coronavirus (CCoV), by blocking viral RNA synthesis. The effect was independent of cyclooxygenase inhibition. The antiviral activity was more than 1000-fold in reducing the virus yield in vivo, in CCoV infected dogs [6]. Indomethacin has the ability to inhibit viral replication and blunt the inflammatory response before the cytokine storm, and hence the potential to improve outcomes. It is inexpensive, widely available and has been successful in treatment of rheumatological conditions. Indomethacin activates double stranded RNA (dsRNA)-dependant protein kinase R (PKR), leading to an interferon and ds-RNA independent phosphorylation of the eukaryotic initiation factor-2 alpha subunit, which shuts off viral protein translation and blocking viral replication [7]. Marinella suggested that indomethacin and resveratrol 
be considered for treatment of or slowing the progression of SARS-CoV-2 infection, given the relative safety, accessibility and low cost [8].

Cough is usually dry, hacking and usually unresponsive to antitussives, in COVID-19. Indomethacin is proven to help in ACE inhibitor induced cough, which could potentially be helpful in treating cough in COVID-19, not responding to usual anti-tussives [9]. The main route of entry of SARS-CoV-2 into the cell is through the ACE2 receptor. The ACE2 function reduces, which releases pro-inflammatory cytokines including kinins [10].

The clinical presentation of COVID-19 infection is similar to that reported in the general population. One of the early reports on COVID-19 in kidney transplant recipients found mortality of $14 \%$ and significant acute kidney injury in 7 patients [11]. Immunosuppressants were reduced and the majority were hospitalised in a study from Belgium. Mortality was $11 \%$ in that cohort, where hydroxychloroquine was used [12].

Fever and cough (78\% and $67 \%$ respectively) were predominant symptoms in that study, whereas it was present in $75 \%$ and $33.3 \%$ respectively, in our study. Only 4 of 22 patients were treated as outpatients in that study, but 8 of 12 patients were managed as outpatients in our study. Extreme lethargy was noted in $91 \%$ of our patients, whereas it was not reported in earlier studies. Four of the patients hospitalized recovered within a median of 12 days (range $=10-28$ days). None in our cohort died. The remaining 8 patients who were treated as outpatients recovered within a median of 4 days (range $=2-15)$. In the study by Devresse, 2 of 18 hospitalized patients died, 13 of them recovered and discharged in a median of 10 days (range $=2-26$ days). Another study by Abolghasemi et al., from Iran, reported on 24 kidney transplant recipients, who were hospitalized for COVID-19. Ten of 24 patients died, and the remaining 14 were discharged in a median of 6.6 days (range $=5-9$ days). They found bilateral multilobar lung involvement in 20 of 24 patients, on CT scan of the chest [3]. Nair V, et al. reported on 10 kidney transplant recipients admitted to 12 centers in New York, USA, with COVID-19. Three of 10 patients died, and the most common chest imaging finding was multifocal patchy pulmonary infiltrates [13].

Overall the kidney transplant recipients in our study had a good outcome, though the sample size was small. The baseline characteristics were similar to other studies, including risk factors like, diabetes mellitus, systemic hypertension and obesity. A study from France found independent association with the following factors in kidney transplant recipients with COVID-19: non-white ethnicity, adjusted odds ratio (OR) 2.17, 95\% confidence interval (95\% CI) 1.23 3.78, obesity (OR, 2.19; 95\% CI 1.19 - 4.05), asthma and chronic pulmonary disease (OR, 3.09; 95\% CI 1.49 - 6.41), diabetes mellitus (OR, 3.33; 95\% CI 1.92 5.77 ) all statistically significant. The mortality was $24 \%$ in that cohort [14]. The low dose and short duration of indomethacin did not cause any significant adverse effects including any untoward rise in serum creatinine. We postulate that, if patients are stable enough to be managed as outpatient, initiating them on indomethacin could reduce the severity of illness, by suppressing the cytokine 
storm. This could also reduce the pressure on hospitals, as hospitalizations may be avoided, if patients remain stable. The antiviral property of indomethacin and its ability to control symptoms like fever and irritative cough, could add to its beneficial effects, with few side effects and low cost.

Limitations of our study include 1) small number of patients included in the study, 2) objective clinical examination of the patients not done which could have identified some findings necessitating hospitalization in some patients, 3) routine blood tests or imaging like like chest x-ray or CT scan not done, which could have identified more severe illness earlier, 4) serial inflammatory markers like CRP, IL-6, ferritin were not done, 5) RT-PCR for SARS CoV2 not rechecked or COVID-19 antibodies not done after clinical recovery, which could have shed more light on their infectivity and immune response. Further studies involving a larger number of kidney transplant recipients with COVID-19, with serial inflammatory markers and radiological studies, followed up over longer periods may help establish a role of indomethacin in not only stable outpatients but also hospitalized patients.

\section{Conclusions}

Kidney transplant recipients are a vulnerable group of patients during the COVID-19 pandemic. They are immunosuppressed and have co-morbidities in addition. Several studies have shown increased mortality in this group. Clinical presentation is similar to that of the general population. Early diagnosis and prompt treatment would likely improve outcomes. The present study shows that low dose indomethacin is likely to improve the outcome, with negligible adverse effects. Larger studies are necessary to establish the role of indomethacin in kidney transplant recipients, in reducing mortality and morbidity.

Ethical clearance was obtained for the study. Informed consent from the patients included in the study to report the case was also obtained.

\section{Conflicts of Interest}

The authors declare no conflicts of interest regarding the publication of this paper.

\section{References}

[1] Abolghasemi, S., Mardani, M., Sali, S., Honarvar, N. and Baziboroun, M. (2020) COVID-19 and Kidney Transplant Recipients. Transplant Infectious Disease, e13413. https://doi.org/10.1111/tid.13413

[2] Nair, V., Jandovitz, N., Hirsch, J.S., et al. (2020) COVID-19 in Kidney Transplant Recipients. American Journal of Transplantation, 20, 1819-1825. https://doi.org/10.1111/ajt.15967

[3] Karuthu, S. and Blumberg, E.A. (2012) Common Infections in Kidney Transplant Recipients. Clinical Journal of the American Society of Nephrology, 7, 2058-2070. https://doi.org/10.2215/CJN.04410512

[4] Alberici, F., Delbarba, E., Manenti, C., et al. (2020) Management of Patients on Di- 
alysis and with Kidney Transplant during SARS-COV-2(COVID-19) Pandemic in Brescia, Italy. Kidney International Reports, 5, 580-585.

https://doi.org/10.1016/j.ekir.2020.04.001

[5] Kute, V., Varugese, S., Prasad, N., Shroff, S. and Agarwal, S.K. (2020) On Behalf of COVID-19 Working Group of Indian Society of Nephrology Renal Transplant Guidelines with Reference to COVID-19 Infection. Indian Journal of Nephrology, 30, 176-178. https://doi.org/10.4103/ijn.IJN_221_20

[6] Amici, C., Di Caro, A., Ciucci, A., et al. (2006) Indomethacin Has a Potent Antiviral Activity against SARS Coronavirus. Antiviral Therapy, 11, 1021-1030.

[7] Amici, C., La Frazia, S., Brunelli, C., Balsamo, M., Angelini, M. and Santoro, M.G. (2015) Inhibition of Viral Protein Translation by Indomethacin in Vesicular Stomatitis Virus Infection: Role of elF2a Kinase PKR. Cellular Microbiology, 17, 1391-1404. https://doi.org/10.1111/cmi.12446

[8] Marinella, M.A. (2020) Indomethacin and Resveratrol as Potential Treatment Adjuncts for SARS-CoV2/COVID-19. International Journal of Clinical Practice, 74, e13535. https://doi.org/10.1111/ijcp.13535

[9] Rudd, P. and Rudd, P. (1992) Indomethacin and Nifedipine Reduced Coughing Induced by Captopril Therapy. ACP Journal Club, 117, 42.

http://www.acpjournals.org/doi/abs/10.7326/ACPJC-1992-117-2-042

[10] Verdecchia, P., Cavallini, C., Spanevello, A. and Angeli, F. (2020) The Pivotal Link between ACE2 Deficiency and SARS-Cov-2 Infection. European Journal of Internal Medicine, 76, 14. https://doi.org/10.1016/j.ejim.2020.04.037

[11] Banerjee, D, Popoola, J., Shah, S., Ster, I.C., Quan, V. and Phanish, M. (2020) COVID-19 Infection in Kidney Transplant Recipients. Kidney International, 97, 1076-1082. https://doi.org/10.1016/j.kint.2020.03.018

[12] Devresse, A., Belkhir, L., Vo, B., et al. (2020) COVID-19 Infection in Kidney Transplant Recipients: A Single Center Case Series of 22 Cases from Belgium. Kidney Medicine, 2, 459-466. https://doi.org/10.1016/j.xkme.2020.06.001

[13] Nair, V., Jandovitz, N., Hirsch, J.S., et al. (2020) COVID-19 in Kidney Transplant Recipients. American Journal of Transplantation, 20, 1819-1825. https://doi.org/10.1111/ajt.15967

[14] Elias, M., Pievani, D., Randoux, C., et al. (2020) COVID-19 Infection in Kidney Transplant Recipients: Disease Incidence and Clinical Outcomes. Journal of the American Society of Nephrology, 31, 2413-2423.

https://doi.org/10.1681/ASN.2020050639 